\title{
Epidemiology of Myosteatosis
}

\author{
Iva Miljkovic, MD, $\mathrm{PhD}^{1}$ and Joseph $\mathrm{M}$ Zmuda, $\mathrm{PhD}^{1}$ \\ ${ }^{1}$ Department of Epidemiology, Graduate School of Public Health, University of Pittsburgh, \\ Pittsburgh, PA, USA
}

\section{Structured Abstract}

Purpose of review-To summarize the epidemiology of myosteatosis and its association with diabetes.

Recent findings-The role of myosteatosis (fat infiltration in skeletal muscle) in diabetes has received considerable attention. There is reasonably consistent evidence that myosteatosis contributes to glucose and insulin abnormalities and diabetes, possibly even independent of overall obesity. Novel hypotheses that link myosteatosis with insulin resistance and type 2 diabetes have also recently been proposed. These hypotheses suggest that impaired secretion of adipokines and/ or modulation of nutritive blood flow to skeletal muscle may be of importance for the development of myosteatosis. Recent longitudinal data also suggests that myosteatosis increases with aging, regardless of changes in body weight.

Summary-Further studies are needed to identify the specific physiological mechanisms that influence myosteatosis, and the mechanisms that link this fat depot with insulin resistance. Longitudinal studies are also needed to evaluate the remodeling of skeletal muscle fat with aging, across a wider age spectrum, and across different populations, especially those at high-risk of developing diabetes. There is also a need to evaluate if myosteatosis influences the incidence of type 2 diabetes independent of overall adiposity. A better understanding of the factors that regulate myosteatosis may lead to the development of novel therapies that influence a more metabolically "healthy" skeletal muscle.

\section{Keywords}

Skeletal muscle; fat; myosteatosis; intramyocellular fat; intermuscular fat; diabetes; insulin resistance; aging

\section{Introduction}

Adipose tissue is considered an endocrine organ that influences numerous physiological and pathological processes. While most individuals with type 2 diabetes (T2D) are overweight or obese, the relationship between excess body fat and impaired glucose homeostasis is still incompletely understood. Excessive body weight or total adiposity, but also the distribution of fat, may determine metabolic risk. During weight gain and with aging, adipocytes can reach their capacity to store fat, which increases ectopic storage of fat around and within the non-adipose tissue organs, such as skeletal muscle, liver, and pancreas. These tissues normally contain only small amounts of fat not intended for long-term lipid storage, but to be used as a source of energy in periods of low glucose supply. There is increasing evidence to suggest that this ectopic fat storage may lead to impaired functions of surrounding tissues

Corresponding Author: Iva Miljkovic, MD, PhD; Department of Epidemiology, Center for Aging and Population Health, 130 North Bellefield Avenue, Room 542, Pittsburgh, PA 15261; Phone: (+1) 412-383-1894; miljkovici@edc.pitt.edu. 
and organs. In the past decade, myosteatosis (the infiltration of fat in skeletal muscle) has emerged as an important fat depot associated with insulin resistance and T2D. The aim of this review is to evaluate and summarize the epidemiology of myosteatosis and its role in T2D.

\section{Review}

\section{Quantification of Myosteatosis}

There are two fat depots within skeletal muscle: fat infiltration within myocytes (intramyocellular fat) and visible fat within the fascia surrounding skeletal muscle (intermuscular fat). The quantification of both skeletal muscle fat depots can be determined by using invasive analyses such as skeletal muscle biopsy samples, or, by using noninvasive radiological techniques such as computed tomography (CT), magnetic resonance imaging (MRI) and magnetic resonance spectroscopy (MRS). Although the accuracy and limitations of these approaches are not the focus of this review, it is important to emphasize potential limitations of the most utilized methods. In particular, one of the limitations of many recent studies is the relatively high coefficients of variation (CVs) of intermuscular fat measurements [1]. This is usually not an issue for intramyocellular fat as their CVs are generally substantially lower than those for intermuscular fat [1]. One of the limitations of the CT technique, utilized frequently in large epidemiological studies, is that it cannot directly measure the lipid content or detect the location of fat storage within myocytes (intramyocellular fat) or lipid droplets surrounding myocytes (extramyocellular fat, which are present in very small quantities) [2]. Most of the studies that utilized CT scans have examined either intermuscular fat or muscle density (also known as muscle attenuation), which is believed to reflect intramuscular fat content. MRS on the other hand, can clearly distinguish between intra- and extra-myocellular fat [2]. Nevertheless, Larson-Mayor et al. have shown that the density of the soleus muscle measured by CT is well correlated with intramyocellular lipid measured by MRS [2]. Goodpaster et al. have shown that the density of the thigh muscle obtained by single-slice CT scan correlates well with the skeletal muscle fat content of percutaneous biopsy specimens [3]. Therefore, despite the aforementioned limitations, both CT and MRS are considered to be reliable methods for assessing skeletal muscle fat content.

\section{Age-related changes in skeletal muscle fat infiltration}

Aging is associated with changes in both total and regional fat distribution. Cross-sectional studies suggest that both intramyocellular and intermuscular fat may increase with advancing age [1]. A proposed mechanism for these alterations involves the possible agerelated change in activation, proliferation and differentiation of quiescent skeletal muscle precursor "stem" cells (i.e., satellite cells) into adipocytes in response to a range of stimuli that occurs with aging [4]. All of the previous studies included relatively small sample sizes and were of cross-sectional design. A study by Delmonico et al, published in October 2009, is the first to examine longitudinal changes in skeletal muscle fat infiltration among older adults. The authors examined changes in CT measures of intermuscular fat over five years in 1678 relatively healthy, higher-functioning men and women of African and European ancestry aged 70-79 years [5]. Intermuscular fat in the mid-thigh increased with aging in both men and women, regardless of weight loss or weight gain or whether participants were weight stable. Percent intermuscular fat increased among all weight change groups in men (35.5\% to $74.6 \%$ ) and women $(16.8 \%$ to $50.0 \%)$. Although this finding indicates that fat infiltration in skeletal muscle is a consistent characteristic of aging, independent of obesity, the participants of this study were relatively healthy and high-functioning. Therefore, additional longitudinal studies are still needed to better understand the natural history, magnitude and patterns of change in skeletal muscle fat infiltration with aging, across a wide 
range of ages and populations recruited without the regard of their health status. A better documentation of changes in skeletal muscle fat distribution with aging may help to identify individuals who are particularly prone to myosteatosis.

\section{Intramyocellular fat and Intermuscular fat: Emerging Risk Factors for Diabetes}

Skeletal muscle accounts for up to $80 \%$ of glucose disposal, and in the rested state, skeletal muscle fatty acid oxidation includes about $90 \%$ of the energy requirements [6]. The main pathophysiological state, observed in more than $90 \%$ of individuals with T2D, is unresponsiveness of myocytes to an insulin stimulus, and consequently impaired glucose uptake into muscle cells [7]. Skeletal muscle is therefore a crucial organ for maintaining glucose homeostasis.

In the past decade, numerous reports described a positive association between intramyocellular fat and insulin resistance. Earlier studies have reported this relationship among obese, older, sedentary lean and diabetic individuals [8], as well as among healthy individuals [9], and offspring of type 2 diabetes patients [10]. The link has not been found among endurance-trained individuals [8], indicating that greater intramyocellular fat is probably a response to the high oxidative requirements during exercise in these individuals. All previous studies were cross-sectional in their design, and included only a small number of participants. Two cross-sectional studies published in the last year, one in 33 obese nondiabetic patients with metabolic syndrome and controls [11], and the other in 54 Caucasians without type 2 diabetes [12], have reported a novel inverse relationship between intramyocellular fat measured by MRS and adiponectin, an adipokine that has antiinflammatory and insulin-sensitizing properties [13].

While most of the earlier studies focused on the metabolic impact of intramyocellular fat, until recently intermuscular fat has not been studied extensively. Goodpaster et al. have reported a positive association between intermuscular fat measured by CT in the mid-thigh and T2D among 2964 elderly men and women [14]. A study conducted in 1249 middle-aged and older men of African ancestry reported intermuscular fat, measured by $\mathrm{CT}$ in the calf, to be positively associated with T2D, independent of total or central obesity, even in a subset of lean men [15]. The same study also suggested that the association between intermuscular fat and T2D may be modified by a family history of T2D [15]. This finding implies that certain individuals may be genetically susceptible to T2D in the presence of greater intermuscular fat. In a recent study of 1623 older men of African and Caucasian ancestry, CT measured intermuscular fat of the calf was greater in men of African ancestry despite their lower total adiposity levels, and positively associated with T2D in both ethnic groups [16]. A study of 249 Caucasian men and women has recently reported that women have considerably less intermuscular fat than men, and that this fat depot is associated with insulin resistance in both genders [17]. A positive association of intermuscular fat with insulin resistance and pro-inflammatory factors (leptin and C-reactive protein (CRP)) [18], and type 2 diabetes $[19,20]$ has been reported in a few other small studies. A recent study of the pro-inflammatory markers, interleukin- 6 (IL-6), CRP, and tumor necrosis factor- $\alpha$ (TNF$\alpha$ ) among 2651 Caucasian and African-American men and women aged 70-79 years participating in the Health $\mathrm{ABC}$ study has reported significant ethnic and gender differences in the association between inflammatory adipokines and intermuscular fat [21]. Higher intermuscular fat was associated with higher concentrations of IL-6 in men of both ethnicities, higher concentrations of CRP in African-American men, and higher concentrations of IL-6, CRP, and TNF- $\alpha$ in Caucasian women [21]. Coen et al. have recently investigated whether the association between intramyocellular fat and insulin resistance is dependent on muscle fiber type in a sample of 22 obese women [22]. They reported a novel and intriguing finding that insulin resistance is associated with greater intramyocellular fat content in type I but not type II myocytes. 
All of these studies were cross-sectional, and many included a small sample size. While these studies consistently support the possibility that both intramyocellular and intermuscular fat are positively associated with insulin resistance and type 2 diabetes, a causal relationship has not yet been established, and remains to be confirmed in larger longitudinal studies. It is of great interest that De Feyter et al. recently reported in their longitudinal study that intramyocellular fat increases the development of T2D in Zucker diabetic fatty rats [23], but such findings are yet to be confirmed in humans. Future studies should also explore the relationship between myosteatosis and incidence of T2D and deterioration of insulin sensitivity, independent of overall adiposity and weight gain. Additionally, as some have previously hypothesized that in addition to impaired cellular mechanisms regulating lipid storage and utilization, an overflow of fat into the intermuscular compartment may be due to a defect in the ability of subcutaneous fat to store excess fatty acids [24], the question of whether fat reduction in the subcutaneous depot or fat accumulation in the intermuscular depot is associated with insulin resistance and type 2 diabetes also remains to be clarified and confirmed in longitudinal studies.

\section{Skeletal Muscle Fat Infiltration and Type 2 Diabetes: Potential Mechanisms}

The mechanisms linking accumulation of fat within skeletal muscle with insulin resistance and type 2 diabetes are still unclear. Some have proposed that accumulation of intramyocellular lipid may impair the insulin receptor substrate 1 (IRS1)/ phosphatidylinositol 3-kinase (PI3K) pathway and growth-factor-regulated protein kinase B (Akt/PKB) pathway of insulin signaling [25]. Others have suggested that increased accumulation of lipid intermediates, such as diacylglycerol, long-chain fatty acyl-CoA species, ceramides, and oxidized lipid mediators, due to increased accumulation of fat in the myocytes, may be responsible for the suppression the insulin signaling [26]. It has been hypothesized that mitochondrial dysfunction in advanced obesity, T2D and aging actually leads to increased intramyocellular fat accumulation [27]. Further studies are clearly needed to clarify whether intramyocellular fat accumulation is a marker or a mediator of insulin resistance.

The mechanisms linking intermuscular fat and insulin resistance are possibly quite different than those underlying the influence of intramyocellular fat on insulin signaling. One theory suggests that the biology of intermuscular adipocytes differ from that of adipocytes from other anatomical sites, though data to support this hypothesis are available only in animal models [28]. One possible mechanism linking intermuscular fat with type 2 diabetes may be through impaired secretion of adipokines [28]. Increased accumulation of intermuscular fat could induce changes in muscle metabolism and insulin sensitivity via local secretion of inflammatory adipokines from fat cells surrounding muscle fibers [28]. Another theory suggests that intermuscular fat may modulate nutritive blood flow to muscle, and thus, contribute to insulin resistance by impairing insulin action and insulin diffusion capacity [29]. However, our understanding of the mechanisms linking skeletal muscle fat infiltration with diabetes, and the reasons why some individuals store more fat in insulin-sensitive tissues than others are currently still very limited.

\section{Skeletal Muscle Fat Infiltration in Obese Individuals}

It has been well documented that skeletal muscle fat infiltration increases in obesity [30]. Various metabolic changes in muscle lipid metabolism occur in obesity, such as reduced fat oxidation and low basal ATP concentrations, leading to increased fat accumulation in skeletal muscle [30]. Malenfant et al. suggested that fat depots in skeletal muscle of obese individuals may be less easily metabolized as fat accumulation is greater in the central region of muscle fibers, where the concentration of mitochondria is lower [31]. Others have proposed that skeletal muscle fat accumulation may be due to a reduction in the utilization 
of fatty acids (i.e. reduced lipolysis and lipid oxidation), increased fatty acid transport and uptake into muscle, or excess lipid availability [32]. Currently, it is thought that there are two different pathways leading to increased intramyocellular fat. The first is a functional adaptation to the increased energy demand in endurance trained athletes. The second is an adaption in conditions of lipid oversupply, including a decrease in fat oxidation, impaired adipogenesis, or a combination of these processes [33]. A recent clinical trial in 16 male healthy offspring of patients with type 2 diabetes and 8 control subjects has shown that fructose overconsumption causes increased skeletal muscle lipid deposition, suggesting that individuals with a family history of T2D may be more prone to develop metabolic disturbances if their diet contains high levels fructose [34]. Shortreed et al. have recently shown that skeletal muscle in mice responds to 8 weeks of high fat diet with an early increase in intramyocellular fat in gastrocnemius/plantaris, but not in soleus [35]. Their findings are of importance as they indicate that high fat diet may induce early adaptive increase of fat infiltration in the skeletal muscle, which occurs in a muscle-specific pattern [35]. Another hypothesis suggests that impaired skeletal muscle mitochondrial function, observed in obesity, leads to impaired fatty acid metabolism, which subsequently results in increased skeletal muscle fat content [25,32]. Nonetheless, whether skeletal muscle fat is simply the consequence of obesity or is a marker of disordered fat partitioning independent of obesity, is still not clear.

A recent study has shown that lean African ancestry non-diabetic men when compared to lean African ancestry diabetic men had significantly greater intermuscular fat, but similar levels of total and central adiposity [15]. Additionally, men of African ancestry may have greater intermuscular fat than Caucasian men, even when matched on age and total body fat [16]. This observation is of particular interest, as differences in body weight, total and central adiposity have failed to explain the excess risk of T2D in African compared with Caucasian ancestry men [36]. Thus, it is possible that independent of overall or central increased adiposity, skeletal muscle fat infiltration may be an important risk factor for T2D in populations of African ancestry, especially among lean individuals. Similarly, lean offspring of parents with T2D have reduced mitochondrial activity and increased intramyocellular fat and insulin resistance, indicating a possible modulating effect of family history of T2D on the development of intramyocellular fat. Further studies are warranted to define the mechanisms for increased fat accumulation in skeletal muscle of lean individuals with T2D. These studies should include populations of different ethnicities.

\section{Conclusion}

Myosteatosis, the infiltration of fat in skeletal muscle, increases with aging and may play a role in the development of type 2 diabetes. Carefully designed and adequately powered longitudinal studies are needed to asses a causal relationship between skeletal muscle fat infiltration and type 2 diabetes, and evaluate the remodeling of skeletal muscle fat with aging, across a wider age spectrum, and between different populations. A better understanding of the factors that regulate fat distribution in skeletal muscle may lead to the development of novel therapies that influence metabolic health.

\section{Acknowledgments}

Dr. Miljkovic is supported by the Mentored Research Scientist Development Award from the National Institute of Diabetes and Digestive and Kidney Diseases (grant K01DK083029). This work was also supported in part by grant R01AR049747 from the National Institute of Arthritis and Musculoskeletal and Skin Diseases.

\section{References and recommended reading}

Papers of particular interest have been highlighted as: 
- of special interest

.. of outstanding interest

1. Kuk JL, Saunders TJ, Davidson LE, et al. Age-related changes in total and regional fat distribution. Ageing Research Reviews. 2009; 8(4):339-348. [PubMed: 19576300]

2*. Larson-Meyer DE, Smith SR, Heilbronn LK, et al. Muscle-associated triglyceride measured by computed tomography and magnetic resonance spectroscopy. Obesity (Silver Spring). 2006; 14(1):73-87. This is the first study to evaluate the correlation between skeletal muscle fat infiltration assessed by CT and MRS, the most widespread techniques in clinical research. [PubMed: 16493125]

3. Goodpaster BH, Kelley DE, Thaete FL, et al. Skeletal muscle attenuation determined by computed tomography is associated with skeletal muscle lipid content. J Appl Physiol. 2000; 89(1):104-10. [PubMed: 10904041]

4. Sinanan ACM, Buxton PG, Lewis MP. Muscling in on stem cells. Biol Cell. 2006; 98(4):203-214. [PubMed: 16545076]

$5 * *$. Delmonico MJ, Harris TB, Visser M, et al. Longitudinal study of muscle strength, quality, and adipose tissue infiltration. Am J Clin Nutr. 2009; 90(6):1579-85. The first study to examine longitudinal changes in skeletal muscle fat infiltration. [PubMed: 19864405]

6. Kelley DE, Mokan M, Simoneau JA, et al. Interaction between glucose and free fatty acid metabolism in human skeletal muscle. J Clin Invest. 1993; 92(1):91-8. [PubMed: 8326021]

7. Saltiel AR, Kahn CR. Insulin signalling and the regulation of glucose and lipid metabolism. Nature. 2001; 414(6865):799-806. [PubMed: 11742412]

8*. Goodpaster BH, He J, Watkins S, et al. Skeletal muscle lipid content and insulin resistance: evidence for a paradox in endurance-trained athletes. J Clin Endocrinol Metab. 2001; 86(12): 5755-61. Cited by 306 articles. First study to point to physical activity as a major determinant of the consequences of increased net muscle lipid uptake. [PubMed: 11739435]

9. Brehm A, Krssak M, Schmid AI, et al. Increased lipid availability impairs insulin-stimulated ATP synthesis in human skeletal muscle. Diabetes. 2006; 55(1):136-40. [PubMed: 16380486]

10. Perseghin G, Scifo P, De Cobelli F, et al. Intramyocellular triglyceride content is a determinant of in vivo insulin resistance in humans: a $1 \mathrm{H}-13 \mathrm{C}$ nuclear magnetic resonance spectroscopy assessment in offspring of type 2 diabetic parents. Diabetes. 1999; 48(8):1600-6. [PubMed: 10426379]

11. Godoy-Matos AF, Bahia LR, Domingues RC, et al. ADIPONECTIN IS RELATED TO INTRAMYOCELLULAR LIPID CONTENT IN NON-DIABETIC ADULTS. J Endocrinol Invest. 2009

12. Kantartzis K, Staiger H, Machann J, et al. Adiponectin oligomers and ectopic fat in liver and skeletal muscle in humans. Obesity (Silver Spring). 2009; 17(2):390-2. [PubMed: 19169222]

13. Wiecek A, Adamczak M, Chudek J. Adiponectin--an adipokine with unique metabolic properties. Nephrol Dial Transplant. 2007; 22(4):981-8. [PubMed: 17234664]

14. Goodpaster BH, Krishnaswami S, Resnick H, et al. Association between regional adipose tissue distribution and both type 2 diabetes and impaired glucose tolerance in elderly men and women. Diabetes Care. 2003; 26(2):372-9. [PubMed: 12547865]

15*. Miljkovic-Gacic I, Gordon CL, Goodpaster BH, et al. Adipose tissue infiltration in skeletal muscle: age patterns and association with diabetes among men of African ancestry. Am J Clin Nutr. 2008; 87(6):1590-1595. This study reported the interaction of family history of diabetes with intermuscular fat, pointing to a possible genetic interaction that increases the risk of diabetes in individuals with high intermuscular fat. [PubMed: 18541544]

16. Miljkovic I, Cauley JA, Petit MA, et al. Greater adipose tissue infiltration in skeletal muscle among older men of African ancestry. J Clin Endocrinol Metab. 2009; 94(8):2735-42. [PubMed: 19454588]

17. Boettcher M, Machann J, Stefan N, et al. Intermuscular adipose tissue (IMAT): association with other adipose tissue compartments and insulin sensitivity. J Magn Reson Imaging. 2009; 29(6): 1340-5. [PubMed: 19422021] 
18. Zoico E, Rossi A, Di Francesco V, et al. Adipose Tissue Infiltration in Skeletal Muscle of Healthy Elderly Men: Relationships With Body Composition, Insulin Resistance, and Inflammation at the Systemic and Tissue Level. J Gerontol A Biol Sci Med Sci. 2009

19. Gallagher D, Kelley DE, Yim JE, et al. Adipose tissue distribution is different in type 2 diabetes. Am J Clin Nutr. 2009; 89(3):807-14. [PubMed: 19158213]

20. Hilton TN, Tuttle LJ, Bohnert KL, et al. Excessive adipose tissue infiltration in skeletal muscle in individuals with obesity, diabetes mellitus, and peripheral neuropathy: association with performance and function. Phys Ther. 2008; 88(11):1336-44. [PubMed: 18801853]

$21 *$. Beasley LE, Koster A, Newman AB, et al. Inflammation and race and gender differences in computerized tomography-measured adipose depots. Obesity (Silver Spring). 2009; 17(5):1062_ 9. First large study to study intermuscular fat in relation to proinflammatory markers. [PubMed: 19165157]

22*. Coen PM, Dube JJ, Amati F, et al. Insulin resistance is associated with higher intramyocellular triglycerides in type I but not type II myocytes concomitant with higher ceramide content. Diabetes. 2010; 59(1):80-8. This study reported that skeletal muscle insulin resistance is related to greater intramyocellular fat content in type I but not type II myocytes. [PubMed: 19833891]

23*. De Feyter HM, Lenaers E, Houten SM, et al. Increased intramyocellular lipid content but normal skeletal muscle mitochondrial oxidative capacity throughout the pathogenesis of type 2 diabetes. FASEB J. 2008; 22(11):3947-55. First longitudinal study of intramyocellular fat, was conducted in Zucker diabetic fatty rats. No such studies exist in humans. [PubMed: 18653763]

24. Gan SK, Samaras K, Thompson CH, et al. Altered myocellular and abdominal fat partitioning predict disturbance in insulin action in HIV protease inhibitor-related lipodystrophy. Diabetes. 2002; 51(11):3163-9. [PubMed: 12401706]

25. Petersen KF, Shulman GI. Etiology of insulin resistance. Am J Med. 2006; 119(5 Suppl 1):S10-6. [PubMed: 16563942]

26**. Timmers S, Schrauwen P, de Vogel J. Muscular diacylglycerol metabolism and insulin resistance. Physiol Behav. 2008; 94(2):242-51. This review discusses the evidence that implicates intermediates in fatty acid metabolism as being central in the development of insulin resistance. [PubMed: 18207474]

27. Lowell BB, Shulman GI. Mitochondrial dysfunction and type 2 diabetes. Science. 2005; 307(5708):384-7. [PubMed: 15662004]

$28 * *$. Vettor R, Milan G, Franzin C, et al. THE ORIGIN OF INTERMUSCULARADIPOSE TISSUE AND ITS PATHOPHYSIOLOGICAL IMPLICATIONS. Am J Physiol Endocrinol Metab. 2009 Excellent review of the origin of intermuscular fat and its pathophysiological implications.

29**. Lee DE, Kehlenbrink S, Lee H, et al. Getting the message across: mechanisms of physiological cross talk by adipose tissue. Am J Physiol Endocrinol Metab. 2009; 296(6):E1210-29. This review discusses the possible novel mechanisms linking skeletal muscle fat infiltration and insulin resistance. [PubMed: 19258492]

30. Goodpaster BH, Theriault R, Watkins SC, et al. Intramuscular lipid content is increased in obesity and decreased by weight loss. Metabolism. 2000; 49(4):467-72. [PubMed: 10778870]

31. Malenfant P, Tremblay A, Doucet E, et al. Elevated intramyocellular lipid concentration in obese subjects is not reduced after diet and exercise training. Am J Physiol Endocrinol Metab. 2001; 280(4):E632-9. [PubMed: 11254471]

32. Roden M. Muscle triglycerides and mitochondrial function: possible mechanisms for the development of type 2 diabetes. Int J Obes (Lond). 2005; 29(Suppl 2):S111-5. [PubMed: 16385762]

$33^{* *}$. Moro C, Bajpeyi S, Smith SR. Determinants of intramyocellular triglyceride turnover: implications for insulin sensitivity. Am J Physiol Endocrinol Metab. 2008; 294(2):E203-13. This review highlights the recently proposed mechanisms of the regulation of skeletal muscle fat infiltration and its turnover. [PubMed: 18003718]

34. Le KA, Ith M, Kreis R, et al. Fructose overconsumption causes dyslipidemia and ectopic lipid deposition in healthy subjects with and without a family history of type 2 diabetes. Am J Clin Nutr. 2009; 89(6):1760-5. [PubMed: 19403641] 
35*. Shortreed KE, Krause MP, Huang JH, et al. Muscle-specific adaptations, impaired oxidative capacity and maintenance of contractile function characterize diet-induced obese mouse skeletal muscle. PLoS One. 2009; 4(10):e7293. This study reports that high fat diet induced early adaptive increase of fat infiltration in the skeletal muscle, which occurs in a muscle-specific pattern. [PubMed: 19806198]

36. Brancati FL, Kao WH, Folsom AR, et al. Incident type 2 diabetes mellitus in African American and white adults: the Atherosclerosis Risk in Communities Study. Jama. 2000; 283(17):2253-9. [PubMed: 10807384] 